\title{
EL VIAJE A ESPAÑA DE COSME LOTTI Y LAS FUENTES DE ROMA, TÍVOLI Y FRASCATI
}

Resulta incuestionable que la presencia de ciertos artífices italianos en la corte de Madrid durante el siglo XVII constituyó un importante y eficaz medio de renovación de los lenguajes artísticos dominantes en la España de entonces. Uno de estos responsables de la introducción de valiosas novedades en el ámbito cortesano fue el polifacético florentino Cosimo o Cosme Lotti. A Lotti se le hizo venir a España en calidad de «fontanero y jardinero», aunque su amplia formación y su destreza en otras disciplinas provocó que en momentos posteriores se le emplease más como escenógrafo teatral y creador de autómatas que como diseñador de jardines. No estaba por

(CARdon, B., ed.), Lovaina, 2001, pp. 289-307 (esp. 303-304). Es de señalar también el hecho de que ambos pudieron ser vecinos. El 13 de septiembre de 1463, quizás tras la muerte de Luis Alimbrot, su hijo Jorge y la madre de éste, Catalina, vendieron una casa colindante con la de "Joanis Reixach pictoris" (BERMEJo, E., "Bartolomé Bermejo. Pintor viajero”, en La pintura gótica hispano flamenca..., p. 101).

${ }^{33}$ La negatividad asociada a la coloración rojiza del cabello es recurso frecuente en la Edad Media. La ostentan algunos de los personajes de reconocida felonía, como Caín, Dalila, Ganelon o Mordred, y, desde la época de Carlos el Calvo, pasa también a ser uno de los rasgos característicos de la iconografía de Judas, compartido, a partir del siglo XIII, con diversas categorías de excluidos como heréticos, musulmanes, vagabundos, leprosos, mendigos y, como no podría ser de otro modo, judíos. Para las connotaciones negativas de determinados colores con relación a la figura de Judas remito al trabajo de Pastoureaux, M., "L'homme roux. Iconographie médiévale de Judas", en Une histoire symbolique du Moyen Age occidental, París, 2004, pp. 197-209.

${ }^{34}$ Es un motivo generalizado en la representación del apóstol traidor al menos desde el siglo XIII. Una circunstancia que responde a una tradición que, desde esa misma centuria, ha tendido a hacer del amarillo un color en franco proceso de devaluación. Es el de los felones y mendaces y, naturalmente, el de los judíos y el de la sinagoga (PASTOUREAUX, ibíd.). Son numerosos los ejemplos que pueden aducirse para la representación de la sinagoga ataviada de amarillo. Por ceñirnos únicamente a lo hispánico señalaremos su presencia en el Cristo Salvator Mundi del Museo del Prado, o en el retablo san Ildefonso de la catedral de Zamora, obras ambas de Fernando Gallego.

35 Algo que, por otra parte, se inscribe en el marco de uno de los argumentos recurrentes de la literatura adversos iudaeos que ya desde san Jerónimo o san Juan Crisóstomo tendió a hacer de Judas la figura emblemática del pueblo hebreo. Cf. Массовy, H., Judas Iscariot and the Myth of the Jewish Evil, Nueva York, 1992, p. 101 y ss.; PAFFENROTH, K., Judas. Images of the Lost Disciple, Louisville, 2001, p. 17 y ss. 
tanto en lo cierto Baldinucci cuando refirió los motivos de la llamada de Lotti a España, pues según él se debió a la petición de Felipe IV al gran duque de un ingeniero capaz de levantar el Coliseo del Buen Retiro, empresa que en realidad no se acometió hasta varios años después de la llegada del maestro a nuestro país ${ }^{1}$.

Don Gaspar de Guzmán, conde-duque de Olivares, tomó como es bien sabido con decisión las riendas del gobierno de España durante su valimiento. La acción de don Gaspar no se limitó al campo de la política, sino que también se ocupó de otros asuntos, tales como la renovación y ampliación de los reales sitios. En este sentido, el principal mérito del conde-duque fue orquestar la veloz construcción del nuevo palacio del Buen Retiro desde comienzos de la década de 1630, aunque ya con anterioridad había emprendido mejoras en las distintas residencias regias. En aquellas reformas que llevó a cabo y en general en todas sus promociones artísticas, el conde-duque tuvo sin dudas muy presentes las experiencias italianas. No ha de obviarse que la niñez de don Gaspar transcurrió en Italia, mientras su padre don Enrique, conde de Olivares, servía a la Corona como embajador y virrey ${ }^{2}$. Como consecuencia de aquella circunstancia personal, el valido tenía un muy buen conocimiento de la cultura italiana, por lo que la importación de modelos y soluciones artísticas ya arraigados en la vecina península que llevó a cabo se basaba en las certezas y convencimientos que le aportaba su propia experiencia.

$\mathrm{Al}$ iniciar distintas reformas en los reales sitios, Olivares debió denotar la falta de ciertos especialistas capaces de materializar sus deseos artísticos para aquellas residencias. Entre los profesionales que seguramente no logró encontrar, y que sin embargo sí existían -y en buen númeroen Italia, estaban los ingenieros hidráulicos o «fontaneros» y también los jardineros especializados. Ante aquella circunstancia, el conde-duque no dudó en solicitar al embajador de España ante la Santa Sede, cargo que entonces ocupaba el duque de Pastrana, que buscase un fontanero de renombre dispuesto a viajar a España para servir a Felipe IV. Aquella solicitud muy probablemente fue cursada durante el año 1625.

Don Ruy Gómez de Silva y Mendoza, tercer duque de Pastrana, fue embajador de Felipe IV en Roma entre 1623 y $1626^{3}$. Don Ruy ya contaba con experiencia previa como diplomático antes de su llegada a Roma, pues había sido embajador extraordinario de Felipe III en París, adonde viajó en 1612 con motivo de las capitulaciones matrimoniales del entonces príncipe de Asturias -el futuro Felipe IV- con Isabel de Borbón y de Luis XIII con Ana de Austria. Algunos años después, en 1619, acompañó al mismo rey durante su jornada en Portugal. Fue a principios de 1623 cuando Felipe IV decidió enviarle a la capital pontificia como su embajador extraordinario ante Gregorio XV, para que interviniese en la compleja disputa de la Valtellina. Su llegada a Roma se produjo en mayo de aquel año ${ }^{4}$, mientras la embajada ordinaria la desempeñaba el duque de Alburquerque. Poco después de su establecimiento en la Ciudad Eterna, falleció al papa Gregorio $\mathrm{XV}$; sin embargo, sus relaciones con Urbano VIII, menos sensible a la causa española que su antecesor, fueron bastante buenas, ya que le conocía desde sus tiempos de cardenal.

En un primer momento, Pastrana consideró la posibilidad de alojarse en el palacio del duque de Zagarolo, para no incomodar así al de Alburquerque ${ }^{5}$, decidiéndose finalmente por alquilar el

\footnotetext{
${ }^{1}$ BALDINUCCI, G.: Notizie de'professori del disegno, Florencia, 1848, vol. V, p. 9.

2 Marañón, G.: El Conde-Duque de Olivares. La pasión de mandar, Madrid, 1999, pp. 45-47.

3 Véase sobre este personaje la obra de Salazar y Castro, L.:, Historia genealógica de la Casa de Silva, donde se refieren las acciones más señaladas de sus señores, las fundaciones de sus mayorazgos y la calidad de sus alianças matrimoniales, Madrid, 1685, vol. II, pp. 565-566.

4 Dadson, T.J.: "Inventario de los cuadros y libros de Ruy Gómez de Silva, III Duque de Pastrana (1626)", Revista de Filología Española, 57,1987, pp. 24-68.

5 Anselmi, A.: Il Palazzo dell'Ambasciata di Spagna presso la Santa Sede, Roma, 2001, p. 201. Aviso de Roma, 30 de septiembre de 1623 .
} 
palacio Gabrielli. Una vez que Alburquerque abandonó Roma, Pastrana se convirtió en embajador ordinario, ocupando la residencia que aquel dejaba libre. Además, gracias a las excelentes relaciones que la Corona mantenía con el gran duque de Toscana, el diplomático fue invitado a trasladarse durante el verano a la Villa Medici ${ }^{6}$, donde sin duda los meses estivos transcurrirían de modo más agradable. Pastrana debió de aceptar la hospitalidad de los Medici de manera recurrente durante su embajada, pues consta que estaba alojado en la Villa en junio de $1626^{7}$, cuando ya se esperaba la llegada de su sucesor y faltaba poco para que emprendiese su regreso a España.

La cordialidad que imperaba entre los Medici y los diplomáticos españoles no sólo se hizo evidente en la generosa oferta que el gran duque hizo a Pastrana, sino que también se manifestó en muchas otras ocasiones. Por ejemplo, consta que el embajador español viajó a Florencia ${ }^{8}$ en el otoño de 1624, asistiendo como invitado de los Medici a una magnífica representación teatral. En octubre de aquel año, el duque presenció la puesta en escena de una obra titulada La Regina Sant'Orsola en el teatro de los Uffizi, con la cuál se quiso honrar al archiduque Carlos de Austria. La escenografía para aquel evento fue diseñada por el afamado artífice Giulio Parigi. Pastrana con seguridad debió quedar gratamente impresionado por el alto nivel que la ingeniería teatral tenía en la corte florentina, pudiendo haber conocido entonces no sólo a Parigi, sino también al polifacético Lotti ${ }^{9}$. Si esta última suposición fuese cierta, no habría de resultar extraño que cuando algún tiempo después el duque recibiese la orden del rey -a través de una carta de Olivares- de reclutar a un artífice italiano capacitado en ingeniería, pensase en Cosme Lotti, quien además de haber dado sobradas muestras en Florencia de su genio, era súbdito de los Medici, por lo que la negociación de su venida a España se vería propiciada por la habitual buena disposición hacia los deseos de la Corona de los señores de la Toscana.

Queda constancia de que en Roma el duque también mantuvo relaciones con diversos artistas. De las cuentas de su embajada en la Ciudad Eterna se desprende que Pastrana contó con los servicios de varios artífices, que por desgracia hoy apenas son conocidos. Entre aquella nómina de artistas vinculados con el duque, se cuentan nada menos que diecisiete plateros, a los que compró o encargó un gran número de piezas de orfebrería ${ }^{10}$. También hubo entre aquellos pintores y probablemente escultores $^{11}$, aunque como consecuencia de las pocas evidencias documentales que se tienen, sus identidades apenas pueden reconstruirse. Serían éstos Giulio y Silvio Capi, Andrea Pelli, Orazio Pacifice, Jacomo Duquesnoy o Jacomo de Hasse, entre otros ${ }^{12}$. No consta por tanto, al menos por el momento, que en Roma el duque tuviese contacto con algún «fontanero» que pudiese responder al perfil profesional que Olivares le demandaba, aunque sí debió de conocer a maestros con cierta habilidad en la materia, puesto que cada año por el día de San Pedro, cuando se celebraba la procesión de la hacanea, se solía instalar una fuente de vino ante la embajada para regocijo del pueblo romano ${ }^{13}$.

Si bien no se tienen pruebas documentales de la presencia de Cosme Lotti en Roma con anterioridad a 1626, ciertas evidencias permiten no obstante suponer que el artista visitó la ciudad antes

\footnotetext{
6 Ibídem, p. 201. Aviso de Roma, 13 de julio de 1624.

7 Ibid., p. 202. Aviso de Roma, 17 de junio de 1626.

8 Santamarina, B.: "Diecisiete plateros italianos y un embajador español. Roma, 1623-1626", Boletín del Museo e Instituto Camón Aznar, 75-76, 1995, pp. 205-220, en especial p. 217, da a conocer la existencia de un libro de cuentas en el que se recogen los gastos privados de Pastrana en Italia; en él, se refieren los que generó el viaje a Florencia. Se conserva en el Archivo Histórico Nacional (AHN), Osuna, cartas, legajo 580.

${ }^{9}$ Así lo sugiere Chaves Montoya, T.: El espectáculo teatral en la corte de Felipe IV. Madrid, 2004.

10 Véase al respecto SANTAMARINA, B.: Ob. cit., pp. 205-220.

11 El duque poseyó una importante pinacoteca, como dio a conocer Dadson, T.J.: Ob. cit., pp. 24-68.

12 Burke, M. y Cherry, P.: Collections of Paintings in Madrid, 1601-1755, Los Ángeles, 1997, I, p. 176.

13 Archivo General de Simancas (AGS), Tribunal Mayor de Cuentas, 2633, Relacion jurada y Quenta de lo que percibió y gastos que executó en las embaxadas ordinaria y extraordinaria cerca de Su Sant[ida]d en Roma, desde 29 de Marzo de 623 h[ast]a 24 de junio de 1626 el Embaxador duque de Pastrana.
} 
de aquel año. Al parecer, el escenógrafo pudo acompañar en una ocasión al secretario granducal Andrea Cioli, quien estaba encargado de negociar ciertos particulares de la unión matrimonial de Vittoria della Rovere con Ferdinando de Medici ${ }^{14}$. Aquellas probables visitas a Roma pudieron permitir algún nuevo encuentro con el duque después de la visita de éste a Florencia en 1624.

Una vez que en la corte de los Medici se hubo decidido que sería Lotti quien viajaría a Madrid para servir a Felipe IV, debió de hacerse necesario que algún agente español se desplazase a Florencia para ultimar los detalles del contrato y entregarle al artífice los anticipos necesarios. Según se desprende de la contabilidad de la embajada de Pastrana, fueron dos los agentes que durante su misión diplomática fueron a Florencia «a cosas del servicio de Su Magestad». Estos agentes fueron don Alonso de Borja, quien tras desembarcar en el puerto de Livorno fue a Florencia y después a Roma, y don Lorenzo de Mendoza, que fue enviado a la capital granducal por orden de Pastrana ${ }^{15}$. No es posible por el momento asegurar cuál de estos dos personajes fue el responsable de ultimar el trato para la venida de Lotti, quien no fue el único artífice florentino en partir entonces para España, ya que vino acompañado por otros tres maestros fontaneros y jardineros, Pier Francesco Gandolfi, Julio de Lorenzo y Julio de Chimendi. En Florencia, Lotti percibió 350 ducados, y Lorenzo y Chimendi, $100^{16}$.

Aunque los documentos relativos a la llegada de Lotti a España que ya son conocidos han desvelado varios aspectos de la presencia en Madrid de Pier Francesco Gandolfi, no se tenía constancia hasta el momento de cierta actividad de este personaje que sin duda debió resultar de una enorme trascendencia para el desarrollo de la jardinería cortesana durante el siglo XVII español. Gandolfi recibió un importantísimo encargo del duque de Pastrana, dibujar las principales fuentes de Roma y sus alrededores. Por orden de Pastrana, Gandolfi tomó «el dibuxo y modelos» de las fuentes de la Ciudad Eterna y de aquellas de Frascati y Tívoli, con la intención de enviarlos a Madrid. Para financiar aquella campaña, se libraron varias partidas para Gandolfi, la mayor de ellas de quinientos julios -la moneda de la Roma de entonces- y una de cien, seguramente destinadas a cubrir los gastos de su viaje, alojamiento y manutención en aquellas cercanas localidades, más otros cincuenta, que tal vez fueron el pago que recibió por aquellos diseños ${ }^{17}$. Esta nueva noticia confirma y precisa la información ofrecida por Baldinucci, quien afirmaba de Gandolfi, «detto il maestrino legnajuolo, uomo di grande ingegno, che, essendo stato a Roma qualche tempo, aveva disegnate e colorite tutte le più belle fontane di quella città» 18 .

Aquello que el conde-duque promovió desde Madrid y que consiguió llevar a cabo con la ayuda de Pastrana en Roma fue una renovación de las fuentes de los reales sitios; llegaron incluso a realizarse en la urbe por el maestro latonero Pietro Guzio las llaves de paso que se necesitaban

\footnotetext{
14 Chaves Montoya, T.: Ob. cit., p. 21.

15 AGS, Tribunal Mayor de Cuentas, 2633, sin foliar.

16 AGS, Tribunal Mayor de Cuentas, 2633, sin foliar. Se procuró igualmente dinero a los florentinos para que lo dejaran en sus casas: "Devenos Su Mag[esta]d al d[i]cho Duque de Pastr[an]a tocante a la d[ic]ha jornada [de Roma] siete mil y trecientos y cinquenta y un Reales en plata q[ue] hazen seiscientos y sesenta y seis d[ucad]o[s] y ciento y ochenta y quatro $\mathrm{m}[\mathrm{a}] \mathrm{r}[$ avedíe]s pagados y gastados con cosme loti florentino yngeniero de fuentes y con los Jardineros sus acompañantes que de orden de Su Mag[esta]d en cartas del S[eño]r Conde Duque se truxeron de Floren[ci]a de mas de quatromil y ciento y treinta y cinco julios que se les socorrieron en florencia para dexar en sus casas que ya van puestos en la quenta sobredicha en la partida decima de gastos extraordinarios".

17 AGS, Tribunal Mayor de Cuentas, 2633, sin foliar: "Quinientos julios que se pagaron a P[ietr $]^{\circ}$ Fran[ces]co Pandolfo natural de Florencia que de orden del d[ic]ho Duque vino a tomar el dibuxo y modelos de las fuentes de Roma y de frascati y de tiboli para llevar a españa las plantas y disegnios de todo a buena quenta de lo q[ue] huviese de haver por su trabajo de la d[ic]ha ocupación" (...) "Cinquenta julios que asimismo se pagaron al d[ic]ho P[ietr] $]^{\circ}$ Fran[ces]co Pandolfo fontanero de florencia a q[uen]ta de los d[ic]hos dibujos de fuentes" (...) "Cient Julios que se pagaron al d[ic]ho Pedro Fran[ces]co Pandolfo fontanero quando fue a Frascati a tomar disignio de las fuentes y a quenta de lo que ha de haver por ellos y por su ocupación".

18 B ALdinUCCI, Notizie..., vol. V, p. 12.
} 
para la instalación de las fuentes ${ }^{19}$. Esto demuestra que la creación de nuevas fuentes o la reparación de las antiguas ya estaba bien planificada, e incluso puesta en marcha por entonces.

El amplísimo catálogo de fuentes públicas y privadas que ofrecía la ciudad de Roma y sus alrededores constituía sin duda uno de los mejores repertorios imaginables para la creación de otras nuevas. La Ciudad Eterna contaba por entonces con una amplia y rica serie de fuentes, la mayor parte construidas en las últimas décadas del siglo $\mathrm{XVI}^{20}$. Aunque aún no se habían levantado las que años más tarde idearía Bernini, verdaderas responsables de la configuración de la imagen de la ciudad histórica, eran ya muy notables las existentes, que en cierta medida respondían a una tradición iniciada en época imperial. Aquella unión de pervivencias imperiales y nuevas propuestas manieristas llegaba al extremo en la localidad de Tívoli, donde podían contemplarse las ruinas de la Villa Adriana y los espectaculares ingenios hidráulicos de la Villa d'Este.

La pequeña localidad de Frascati, situada a unos veinte kilómetros al sureste de Roma, comenzó a ser frecuentada como lugar de retiro y veraneo de importantes dignatarios de la Iglesia a raiz de las estancias que el Papa Paulo III tuvo allí desde 1536. De forma progresiva, distintos personajes destacados de la corte pontificia fueron construyendo sus villas en los alrededores de Frascati, hasta configurarse once suntuosísimas residencias en la década de 162021. Pastrana también se desplazó en ciertas ocasiones a la localidad de Frascati22. Se sabe por los Avvisi de Roma, que el diplomático fue invitado por el cardenal Sforza a su villa, siendo también agasajado en las otras principales residencias, como en la del cardenal Borghese ${ }^{23}$ (Fig. 1). Consta también cómo durante su embajada, al menos en una ocasión, un enviado del duque fue a Frascati a darle la enhorabuena a algunos de los cardenales que en ocasiones allí se retiraban ${ }^{24}$.

Las fuentes de las villas de Frascati eran por entonces unas de las más elevadas muestras de combinación de técnicas hidráulicas e invención artística que podían verse en Italia. Entre las residencias que la nobleza romana fue construyendo en aquella localidad, destacaba por su monumentalidad y por la belleza de sus jardines la de los Aldovrandini, cuyas fuentes habían sido realizadas con la intervención de Orazio Olivieri, quien años atrás cuidó las instalaciones hidráulicas de la Villa d'Este en Tívoli.

En el parque de aquella villa podían contemplarse órganos de agua, autómatas, animales recreados y otros prodigios 25 . Sobresalía entre todas aquellas creaciones el llamado Teatro dell'Acqua, espectacular simbiosis de arquitectura y escultura ideada por Carlo Maderno ${ }^{26}$ (Fig. 2). La compleja iconografía del Teatro contaba con elementos simbólicos muy cercanos a la Casa de

\footnotetext{
19 AGS, Tribunal Mayor de Cuentas, 2633, sin foliar.

20 Sobre las fuentes de Roma, véase CALlaRi, L.: Le fontane di Roma, Roma, 1945; D’OnofRIo, C.: Le fontane di Roma, Roma, 1986.

${ }^{21}$ Las once villas eran Mondragone, Tusculana, Borghese, Ruffina, Ruffinella, Aldobrandini, Sora, Altemps, Belpoggio, Acquaviva y Arrigone; véase sobre ellas Coffin, D.R.: The Villa in the Life of Renaissance Rome, Princeton, 1988, en especial pp. 40 y ss; EHRLICH T.L.: Landscape and Identity in Early Modern Rome: Villa Culture at Frascati in the Borghese Era, Cambridge, 2002; Franck, C.: Die Barockvillen in Frascati, Munich, 1956; MARCuCCI, L.: "Villa Mondragone a Frascati", Quaderni dell'Istituto di Storia dell'Architettura, 169/174, 1982, pp. 117-136; D’ONofRIo, C.: La Villa Aldobrandini di Frascati, Roma, 1963; GUERRIERI Borsol, M.B.: Villa Sora a Frascati, Roma, 2000; idem: Villa Belpoggio a Frascati, Roma, 1997.

22 Así lo demuestra una carta enviada por el duque al rey desde Frascati, conservada en la Real Biblioteca, II/2197, doc. $n^{\circ}$ 9. En ella se trata del complejo asunto de la Valtellina.

23 Anselmi, A.: Ob. cit., p. 200. Aviso de Roma, 3 de junio de 1623.

24 AGS, Tribunal Mayor de Cuentas, 2633, sin foliar: "Setenta y siete julios que gastó un gentilhombre con los cavallos y cocheros en yr a Frascati a dar la enhorabuena a tres cardenales".

25 TAgliolinI, A.: Storia del giardino italiano. Gli artisti, l'invenzione, le forme dall'antichità al XIX secolo, Florencia, 1988 , p. 238.

${ }^{26}$ Sobre la intervención de este arquitecto en la villa, véase HiBBARD, H.: Carlo Maderno, Milán, 2001, pp. 164-171.
} 
Austria, como las columnas de Hércules o el Atlas sosteniendo la esfera celeste ${ }^{27}$. No todas las fuentes de las villas de Frascati que por entonces existían han llegado hasta el presente, si bien buen número de ellas pueden ser rememoradas gracias a los grabados que muchas décadas después, en 1675, realizó Giovan Battista Falda ${ }^{28}$.

Los nobles y religiosos españoles que estuvieron presentes en Roma desde mediados del siglo XVI sin duda debieron de tener numerosas oportunidades de conocer las villas que en Frascati y Tívoli iban construyendo los cardenales y aristócratas de la corte pontificia. Pero además de por el conocimiento directo de algunos pocos privilegiados, en Madrid también se pudo saber cómo eran las villas y las fuentes de Roma y sus alrededores a través de las descripciones que de ellas se incluían en algunos libros y de los grabados que los ilustraban ${ }^{29}$. No obstante, gracias a la oportuna orden que Pastrana dio a Gandolfi, en la corte española se contó con los servicios de un maestro que conocía de primera mano las principales obras de fontanería toscanas y romanas.

En diversas ocasiones, el duque de Pastrana había solicitado al rey licencia para volver a España, ya que se venía resintiendo de su quebrantada salud. No fue hasta 1626 cuando llegó el anhelado permiso real para abandonar la embajada. Pastrana debía permanecer en Roma hasta ser relevado por quien había sido designado para sucederle, el conde de Oñate. Puede que fuera por una simple coincidencia, pero parece que el viaje de Lotti desde Florencia a Roma se hizo coincidir con las fechas inmediatas a la partida del duque, de tal modo que ambos pudieran viajar juntos a España. El maestro salió de la capital granducal el 23 de junio de $1626^{30}$, aunque todo indica que Gandolfi había llegado a la Ciudad Eterna tiempo antes que Lotti, quien lo haría en los primeros días de julio de 1626.

A mediados de junio de 1626 llegó al puerto de Civitavecchia don Íñigo Vélez de Guevara y Tassis, V conde de Oñate. El conde fue alojado en un primer momento en la Villa Medici, ya que se preveía entonces la inminente partida de Pastrana de Roma. Desde luego, el duque no abandonaría la ciudad de inmediato, sino que aún debió permanecer en ella el tiempo necesario para preparar su regreso a España y el de su familia. Finalmente, partió de Roma poco después del 20 de junio de 1626, habiendo recibido numerosos presentes del Sumo Pontífice ${ }^{31}$. Es probable que el duque y Gandolfi se reunieran con los otros tres florentinos en Civitavecchia, donde habrían embarcado con destino a algún puerto del Levante español. Sea como fuere, lo cierto es que los florentinos viajaron a España con Pastrana y su séquito, como confirman ciertos documentos que aseguran que a Lotti lo «traxo el dicho Duque consigo el año pasado de [1]626»32.

Una vez arribados a las costas españolas, continuarían el viaje hasta Madrid. Pastrana viajó a España estando enfermo, y también Lotti padeció durante el trayecto, pues se rompió una pierna antes de llegar a destino. El 8 de septiembre de 1626 entraron en la corte, y ante la imposibilidad del duque para acompañar al maestro ante Olivares, Lotti acabó presentándose al valido con un

\footnotetext{
27 Steinberg, R. M.: “The iconography of the Teatro dell'Acqua at the Villa Aldobrandini”, The Art Bulletin, 47, 1965, pp. 453-469.

28 Le Fontane di Roma (...) disegnate, et intagliate da Gio. Battista Falda, Roma, Gio. Giacomo de Rossi, 1675. Existe un ejemplar de esta rara colección de grabados en la Biblioteca Nacional, ER/1940.

${ }^{29}$ Me refiero a los libros de MAGgIO, G., Fontane diverse che si vedono nel'Alma cittá di Roma et altre parti d'Italia. Roma, 1618 y Del Re, A.: Dell'antichità tiburtine (...) nel quale si deferivono le maraviglie del palazzo, \& giardino della serenissima famiglia d'Este, \& loro fontane, e statue, Roma, Giacomo Mascardi, 1611.

30 Así consta en un documento redactado con motivo de un aumento de su salario en 1628, estando ya en España; véase Archivo General de Palacio (AGP), Registro, Cédulas Reales, XII, 15/10/1628. Publicado por MARTínEZ LEIVA, G.: "En torno a Cosme Lotti: nuevas aportaciones documentales", Madrid. Revista de arte, geografía e historia, 3,2000 , p. 335.

31 Anselmi, A.: Ob. cit., p. 202. Aviso de Roma, 26 de junio de 1626.

32 Shergold, N.D.: "Documentos sobre Cosme Lotti, escenógrafo de Felipe IV", en Studia Iberica. Festschrift für Hans Flasche, Berna y Munich, 1973, pp. 589-602, en particular p. 596.
} 
Fig. 1. Giovan Battista Falda. Teatro de las fuentes de la villa Borghese-Mondragone en Frascati.

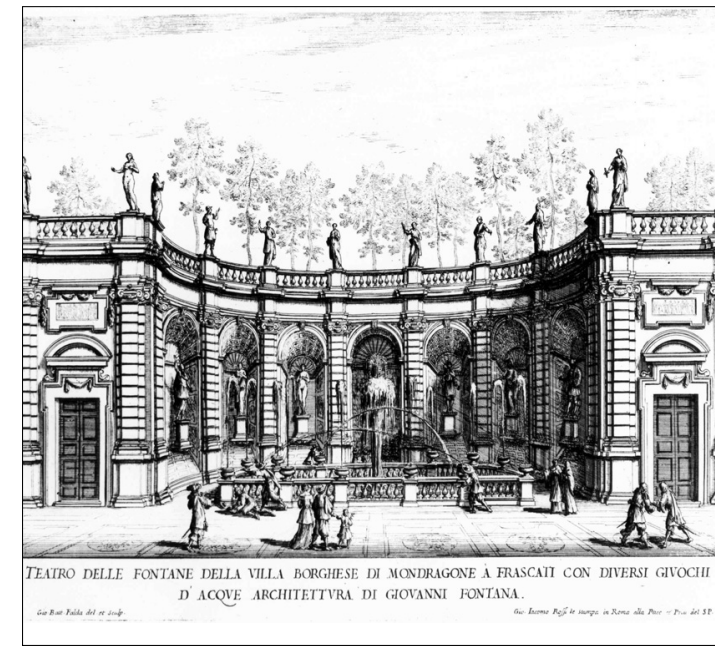

Fig. 2. Giovan Battista Falda. Cascada sobre el Teatro del Agua de la Villa Aldovrandini en Frascati.
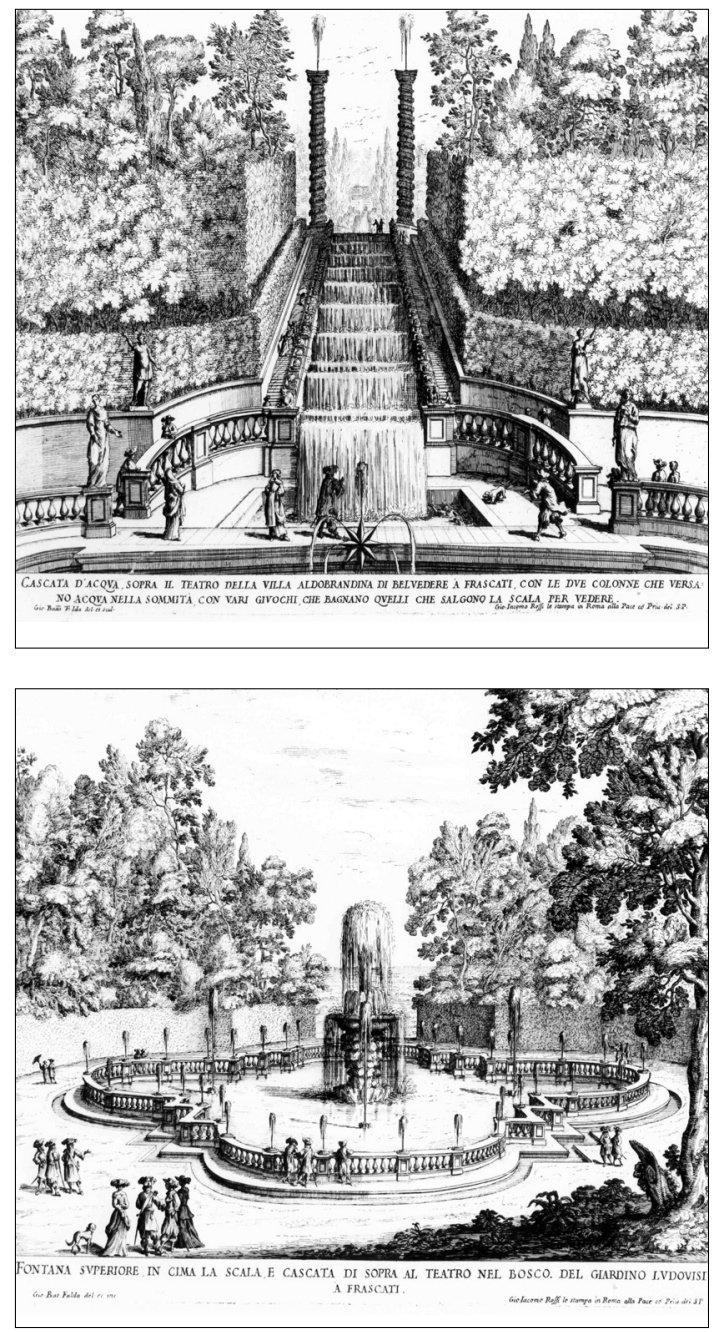

Fig. 3. Giovan Battista Falda. Fuente superior del
Teatro del bosque de la Villa Ludovisi en Frascati. 
billete suyo ${ }^{33}$. Don Ruy no logró superar la enfermedad que por entonces le atormentaba, pues falleció pocos meses después, el 23 de diciembre de 1626.

Es de suponer que Lotti y su equipo comenzaran a trabajar en cuanto hubieran recibido instrucciones del conde-duque. Al maestro se le alojó en la Casa de Campo, tal vez el primer sitio en el que debía instalar nuevas fuentes. Gandolfi también permaneció en Madrid, mientras que los dos jardineros fueron enviados a Aranjuez ${ }^{34}$. Si se prescindió en Madrid de los servicios de los dos jardineros, tenía que ser porque aquello realmente necesario entonces era el diseño, instalación y arreglo de las fuentes de los reales sitios, y no sólo del Alcázar y la Casa de Campo, sino también de El Pardo y La Zarzuela ${ }^{35}$. En aquella operación se debieron de usar como modelos los dibujos que Gandolfi había realizado en Roma y sus alrededores, los cuáles no parece que quedasen en manos de Pastrana ${ }^{36}$, sino que o bien los conservaron los propios artistas, o pasaron a los intendentes de la Real Casa.

La variedad y la originalidad de las fuentes construidas por aquellos años en los reales sitios sin duda fueron consecuencia, al menos en parte, del conocimiento y las habilidades de Lotti y Gandolfi. Y desde luego, una vez que la idea de crear el nuevo palacio del Buen Retiro en Madrid comenzó a hacerse realidad, la experiencia de Lotti con seguridad fue de enorme ayuda. Gandolfi ya no se encontraba por entonces en Madrid, puesto que en 1629, aludiendo problemas de salud, consiguió licencia para regresar a su patria ${ }^{37}$. Un año antes, en 1628, Lotti había podido afianzar su posición en el complejo mundo de la corte, obteniendo un considerable aumento de sueldo por parte del rey ${ }^{38}$, incremento que sin embargo no le libraría en los años sucesivos de atravesar etapas de penuria económica.

Los diseños de las fuentes de Roma, Tívoli y Frascati que se trajeron a Madrid por orden del duque de Pastrana debieron de servir para inspirar la grandiosa creación del Buen Retiro. El conocimiento de las fuentes que ornaban sus jardines dista aún hoy de ser completo, pero igualmente pueden reconocerse ciertos paralelismos entre algunos de los espacios más carismáticos del parque del Buen Retiro y ciertas fuentes de Roma, Tívoli o Frascati. Sorprende por ejemplo el parecido de una de las fuentes del Giardino Ludovisi en Frascati (Fig. 3) y el original estanque ochavado del Retiro, e incluso podría pensarse en cierta inspiración de la ermita de San Antonio, rodeada de un peculiar foso, en el Teatro Marítimo de la Villa Adriana en Tívoli. No obstante, sería exagerado atribuir a Lotti el diseño integral de los jardines del Retiro, pues como muy oportunamente apuntaron Brown y Elliott, Giovan Battista Crescenzi tuvo una intervención destacada en aquella misma empresa ${ }^{39}$. En cualquier caso, cabe una vez más reivindicar el papel desempeñado por Cosme Lotti, y también por su menos conocido ayudante Pier Francesco Gandolfi en la introducción de importantes novedades en el diseño de fuentes y jardines en la corte de Madrid, confirmando la opinión de Baldinucci, quien no dudó en calificar al maestro de «uno dei più bizzarri ingegni del suo tempo» 40 .

David García Cueto Universidad de Granada

\footnotetext{
33 Así consta en una carta enviada por un diplomático florentino entonces destacado en Madrid a la corte granducal; véase Whitaker, S.B.: "Florentine Opera comes to Spain: Lope de Vega's La Selva sin amor", Journal of Hispanic Filology, 9, 1984, pp. 43-66, en especial pp. 49 y 62.

34 Brown, J. y Elliott, J.: Un palacio para el rey. El Buen Retiro y la corte de Felipe IV. Madrid, 2003, p. 46.

35 Refuerza esta idea con documentación inédita Zangheri, L.: "Artisti toscani per la corte di Spagna", Antichità Viva, 35, 1996, pp. 14-20, en especial p. 19.

36 Dadson, T.J.: Ob. cit. Ningún registro que pueda identificarse con aquellos diseños se encuentra en el inventario póstumo de los bienes del duque.

37 Martínez Leiva, G.: Ob. cit. Véase en especial el documento ${ }^{\circ} 4$.

38 Morán Turina, M. y Checa Cremades, F.: Las Casas del Rey. Casas de Campo, Cazaderos y Jardines. Siglos XVI y XVII. Madrid, 1986, p. 142, nota 58.

39 Brown, J. y Elliott, J.: Ob. cit., vol. V, p.7.

${ }^{40}$ Baldinucci, G.: Ob. cit., vol. V, p. 7.
} 